\title{
Remarks on endpoints of multivalued mappings in geodesic spaces
}

Satit Saejung*

\section{"Correspondence:}

saejung@kku.ac.th

Department of Mathematics,

Faculty of Science, Khon Kaen

University, Khon Kaen, 40002,

Thailand

Research Center for Environmental

and Hazardous Substance

Management, Khon Kaen University,

Khon Kaen, 40002, Thailand

\begin{abstract}
We discuss Panayanak's results on the existence of an endpoint of a multivalued nonexpansive mapping. We show that all of his results can be extended and some can be established in a wider class of mappings. Out of his three open questions, two of them are solved in affirmative.
\end{abstract}

\section{Introduction}

Let $(X, d)$ be a metric space. The distance from an element $x \in X$ to a nonempty subset $E \subset X$ is defined by

$$
d(x, E):=\inf \{d(x, y): y \in E\}
$$

For a nonempty subset $E \subset X$, we denote by $\mathcal{B C}(E)(\mathcal{K}(E)$, respectively) the family of nonempty bounded and closed subsets (nonempty compact subsets, respectively) of $E$. Note that $\mathcal{K}(E) \subset \mathcal{B C}(E)$ and the inclusion may be proper. The Pompeiu-Hausdorff distance on $\mathcal{B C}(X)$ is defined by

$$
H(A, B):=\max \left\{\sup _{a \in A} d(a, B), \sup _{b \in B} d(b, A)\right\}
$$

for all $A, B \in \mathcal{B C}(X)$.

An element $x \in E$ is a fixed point of a multivalued mapping $T: E \rightarrow \mathcal{B C}(X)$ if $x$ belongs to the set $T x$. Moreover, if $\{x\}=T x$, then $x$ is called an endpoint of $T$ (or a stationary point of $T$ ). The set of all endpoints (all fixed points, respectively) of $T$ is denoted by $\operatorname{End}(T)$ $(\operatorname{Fix}(T)$, respectively). It is clear that $\operatorname{End}(T) \subset \operatorname{Fix}(T)$ and the inclusion may be proper (see Example 1). In the case of single-valued mappings, both notions coincide.

The existence of an endpoint and of a fixed point of a multivalued mapping has been widely investigated by many researchers (see, e.g., [1-8]). Corley [1] proved that a maximization with respect to a cone, which subsumes ordinary and Pareto optimization, is equivalent to the problem of finding an endpoint of certain multivalued mapping. Note that the results in multivalued case are suggested but do not follow directly from the one in the single-valued case. In spite of the Michael selection theorem, which gives a continuous selection for multivalued upper semicontinuos mappings, almost nothing is known

(c) 2016 Saejung. This article is distributed under the terms of the Creative Commons Attribution 4.0 International License (http://creativecommons.org/licenses/by/4.0/), which permits unrestricted use, distribution, and reproduction in any medium, provided you give appropriate credit to the original author(s) and the source, provide a link to the Creative Commons license, and indicate if changes were made. 
about obtaining a nonexpansive selection. In our problem studied below, we do not know how they can be proved via the classical results for single-valued mappings.

First, let us recall the following simple example of Ko [9]. It suggests that fixed point results in the single-valued case should be extended to endpoint results in the corresponding multivalued one.

Example 1 Let $X$ be the two-dimensional Euclidean space $\left(\mathbb{R}^{2},\|\cdot\|\right)$, and $E=[0,1] \times[0,1]$. Let $T: E \rightarrow \mathcal{K}(E)$ be defined by

$$
T(a, b):=\text { the closed convex hull of }\{(0,0),(a, 0),(0, b)\} .
$$

Note that $H(T(a, b), T(c, d)) \leq\|(a, b)-(c, d)\|$ for all $(a, b),(c, d) \in E$. Moreover, we remark the following facts.

- It is clear that $\operatorname{Fix}(T)=\{(a, b) \in E: a b=0\}$ and $\operatorname{End}(T)=\{(0,0)\}$. Hence, $\operatorname{End}(T)$ is convex, but $\operatorname{Fix}(T)$ is not.

- This example shows that even in a Hilbert space the class of $\Gamma$-type mappings, which is given analogously to Bruck's theorem does not include all nonexpansive mappings (see [5], Example 5.1, for more details).

- For a fixed element $\left(a_{0}, b_{0}\right) \in E$ and $t \in(0,1)$, define $S_{t}: E \rightarrow \mathcal{K}(E)$ by

$$
S_{t}(a, b):=(1-t)\left(a_{0}, b_{0}\right)+t T(a, b) .
$$

It follows that, as $t \uparrow 1$, the net $\left\{\operatorname{Fix}\left(S_{t}\right)\right\}_{t \in(0,1)}$ does not converge to the fixed point of $T$ nearest to $\left(a_{0}, b_{0}\right)$ even in the weaker convergence of sets (see [6]).

Recall that a multivalued mapping $T: E \rightarrow \mathcal{B C}(X)$ has the approximate endpoint property if

$$
\inf _{x \in E} \sup _{y \in T x} d(x, y)=0 .
$$

Obviously, if $\operatorname{End}(T) \neq \varnothing$, then $T$ has the approximate endpoint property. In this paper, we investigate the following statement:

$T$ has the approximate endpoint property $\quad \Longrightarrow \quad \operatorname{End}(T) \neq \varnothing$.

This research problem has been investigated by many mathematicians (see [10-13] for the single-valued case and $[5,9,14]$ for the multivalued one).

Recently, Panyanak [15] showed that (A) holds in some situations. We first quote all main results recently proved in his paper. Some relevant definitions and concept will be given in the next section.

Theorem P1 Suppose that $E$ is a nonempty bounded closed convex subset of a uniformly convex Banach space X. If $T: E \rightarrow \mathcal{K}(E)$ is a nonexpansive mapping, then (A) holds.

Theorem P2 Suppose that $E$ is a nonempty bounded closed convex subset of a reflexive Banach space $X$ with the Opial property. If $T: E \rightarrow \mathcal{K}(X)$ is a nonexpansive mapping, then (A) holds. 
Theorem P3 Suppose that $E$ is a nonempty bounded closed convex subset of a complete $\mathrm{CAT}(0)$ space $X$. If $T: E \rightarrow \mathcal{K}(X)$ is a nonexpansive mapping, then (A) holds.

Note that the proofs of Theorems P1, P2, and P3 are based on the technique of asymptotic center introduced by Lim [16].

Theorem P4 Suppose that $E$ is a nonempty convex subset of a complete $\mathrm{CAT}(0)$ space $X$. If $T: E \rightarrow \mathcal{K}(X)$ is a nonexpansive mapping, then $\operatorname{End}(T)$ is convex.

We also quote some of his questions.

Question P1 Let $E$ be a nonempty bounded closed convex subset of a uniformly convex Banach space $X$, and $T: E \rightarrow \mathcal{K}(X)$ be a nonexpansive mapping. Does (A) hold?

Question P2 Let $E$ be a nonempty closed convex subset of a uniformly convex Banach space $X$, and $T: E \rightarrow \mathcal{B C}(X)$ be a nonexpansive mapping. Is $\operatorname{End}(T)$ convex?

The purpose of this paper is to give significant extensions of all theorems above. We also give affirmative answers to the preceding two questions. It should be noted that our proofs are different from those in Panyanak's paper. Moreover, many results are established under weaker assumptions.

\section{Main results}

For an element $x$ in a metric space $X:=(X, d)$ and for a nonempty bounded subset $E$ of $X$, we write

$$
D(x, E):=\sup \{d(x, y): y \in E\} .
$$

Using this notation, for any mapping $T: E \rightarrow \mathcal{B C}(X)$, we have the following statements:

- $T$ has an end point if and only if there exists an element $x \in E$ such that $D(x, T x)=0$.

- $T$ has the approximate endpoint property if and only if there exists a sequence $\left\{x_{n}\right\}$ in $E$ such that $D\left(x_{n}, T x_{n}\right) \rightarrow 0$.

Recall that a multivalued mapping $T: E \rightarrow \mathcal{B C}(X)$ is nonexpansive if it does not increase the distances, that is,

$$
H(T x, T y) \leq d(x, y) \quad \text { for all } x, y \in E .
$$

Let us first start with the following easy observation.

Proposition 2 Let $E$ be a nonempty subset of a metric space $X$, and $T: E \rightarrow \mathcal{B C}(X)$ be given. Let $x, y \in E$. Then the following statements hold.

- If $y^{\prime} \in T y$, then $d\left(x, y^{\prime}\right) \leq D(x, T x)+H(T x, T y)$.

- If $y^{\prime} \in T y$ and $T$ is nonexpansive, then $d\left(x, y^{\prime}\right) \leq D(x, T x)+d(x, y)$.

Proof We prove only the first assertion. Let $x, y \in E$, and $x^{\prime} \in T x$ and $y^{\prime} \in T y$. It follows that

$$
d\left(x, y^{\prime}\right) \leq d\left(x, x^{\prime}\right)+d\left(x^{\prime}, y^{\prime}\right) \leq D(x, T x)+d\left(x^{\prime}, y^{\prime}\right) .
$$


Since $x^{\prime} \in T x$ is arbitrary, we have

$$
d\left(x, y^{\prime}\right) \leq D(x, T x)+d\left(y^{\prime}, T x\right) \leq D(x, T x)+H(T y, T x) .
$$

This completes the proof.

The following result follows easily from the preceding proposition.

Lemma 3 Let $E$ be a nonempty subset of a metric space $X$, and $T: E \rightarrow \mathcal{B C}(X)$ be a continuous mapping. If $\left\{u_{n}\right\}$ is a sequence in $E$ such that $\lim _{n} D\left(u_{n}, T u_{n}\right)=0$ and $\left\{u_{n}\right\}$ converges to some element $u \in E$, then $u \in \operatorname{End}(T)$.

Proof Let $u^{\prime} \in T u$. It follows from Proposition 2 that $d\left(u_{n}, u^{\prime}\right) \leq D\left(u_{n}, T u_{n}\right)+H\left(T u, T u_{n}\right)$. Since $T$ is continuous, we have $\lim _{n} H\left(T u, T u_{n}\right)=0$. Then the sequence $\left\{u_{n}\right\}$ converges to $u^{\prime}$, and hence $u=u^{\prime} \in T u$. This finishes the proof.

\subsection{Endpoint results in strictly convex spaces and uniformly convex spaces}

A Banach space $X$ is strictly convex if the following implication holds:

$$
\|u\| \leq 1, \quad\|v\| \leq 1, \quad\|u-v\|>0 \quad \Longrightarrow \quad \frac{1}{2}\|u+v\|<1 .
$$

The uniform version of this property is as follows: $X$ is uniformly convex [17] if for each $\varepsilon>0$, there exists $\delta>0$ such that

$$
\|u\| \leq 1, \quad\|v\| \leq 1, \quad\|u-v\| \geq \varepsilon \quad \Longrightarrow \quad \frac{1}{2}\|u+v\| \leq 1-\delta .
$$

Every uniformly convex space is strictly convex. The converse is not true (see [18], Example 5.2.13).

First, we give an affirmative answer to Question P2. Moreover, we show that the uniform convexity can be weaken to the strict convexity. The following lemma seems to be known, but we give a proof for completeness.

Lemma 4 If $u$ and $v$ are two elements in a strictly convex space such that $\|u+v\|=\|u\|+$ $\|v\|$, then $\|v\| u=\|u\| v$.

Proof If either $u=0$ or $v=0$, then the conclusion holds. We now assume that $u \neq 0$ and $v \neq 0$. Let $t=\|u\| /(\|u\|+\|v\|)$. Then $t \in(0,1)$. Since $\|u+v\|=\|u\|+\|v\|$, we have

$$
\left\|t \frac{u}{\|u\|}+(1-t) \frac{v}{\|v\|}\right\|=1
$$

Without loss of generality, we may assume that $t \leq 1 / 2$. (Otherwise, let $t=\|v\| /(\|u\|+\|v\|)$.) It follows then that

$$
\begin{aligned}
1 & =\left\|t \frac{u}{\|u\|}+(1-t) \frac{v}{\|v\|}\right\|=\left\|2 t\left(\frac{1}{2} \frac{u}{\|u\|}+\frac{1}{2} \frac{v}{\|v\|}\right)+(1-2 t) \frac{v}{\|v\|}\right\| \\
& \leq 2 t\left\|\frac{1}{2} \frac{u}{\|u\|}+\frac{1}{2} \frac{v}{\|v\|}\right\|+1-2 t .
\end{aligned}
$$


In particular,

$$
\frac{1}{2}\left\|\frac{u}{\|u\|}+\frac{v}{\|v\|}\right\|=1
$$

Since the space is strictly convex, we have

$$
\frac{u}{\|u\|}=\frac{v}{\|v\|}
$$

This completes the proof.

Theorem 5 Let E be a nonempty closed convex subset of a strictly convex Banach space $X$. If $T: E \rightarrow \mathcal{B C}(X)$ is a nonexpansive mapping, then $\operatorname{End}(T)$ is convex.

Proof Let $u, v \in \operatorname{End}(T)$ and $z=(1-t) u+t v$ where $t \in(0,1)$. We may assume that $u \neq v$. To see that $z \in \operatorname{End}(T)$, let $z^{\prime} \in T z$. It follows from Proposition 2 that

$$
\left\|u-z^{\prime}\right\| \leq\|u-z\| \quad \text { and } \quad\left\|v-z^{\prime}\right\| \leq\|v-z\| .
$$

We consider the following:

$$
\begin{aligned}
\|u-v\| & =\left\|\left(u-z^{\prime}\right)+\left(z^{\prime}-v\right)\right\| \\
& \leq\left\|u-z^{\prime}\right\|+\left\|z^{\prime}-v\right\| \\
& \leq\|u-z\|+\|z-v\| \\
& =t\|u-v\|+(1-t)\|u-v\| \\
& =\|u-v\| .
\end{aligned}
$$

It follows from Lemma 4 that

$$
\left\|z^{\prime}-v\right\|\left(u-z^{\prime}\right)=\left\|u-z^{\prime}\right\|\left(z^{\prime}-v\right) .
$$

Moreover,

$$
\begin{aligned}
& \left\|z^{\prime}-v\right\|=\|z-v\|=(1-t)\|u-v\|, \\
& \left\|u-z^{\prime}\right\|=\|u-z\|=t\|u-v\| .
\end{aligned}
$$

Hence, $(1-t)\left(u-z^{\prime}\right)=t\left(z^{\prime}-v\right)$, that is, $z=(1-t) u+t v=z^{\prime} \in T z$. This completes the proof.

We simultaneously extend Theorem P1 and give an affirmative answer to Question P1. We also introduce the following concept, which is a multivalued version of [19].

Definition 6 Let $E$ be a nonempty convex subset of a Banach space $X$. A multivalued mapping $T: E \rightarrow \mathcal{B C}(X)$ is of convex type if $\lim _{n} D\left(z_{n}, T z_{n}\right)=0$ whenever $\left\{u_{n}\right\}$ and $\left\{v_{n}\right\}$ are sequences in $E$ such that $\lim _{n} D\left(u_{n}, T u_{n}\right)=\lim _{n} D\left(v_{n}, T v_{n}\right)=0$ and $z_{n}=\frac{1}{2} u_{n}+\frac{1}{2} v_{n}$ for all $n \geq 1$. 
In the presence of uniform convexity, this class of mappings includes all nonexpansive ones. Note that $X$ is uniformly convex if and only if $\lim _{n}\left\|x_{n}-y_{n}\right\|=0$ whenever $\left\{x_{n}\right\}$ and $\left\{y_{n}\right\}$ are sequences in $X$ satisfying $\lim _{n}\left\|x_{n}\right\|=\lim _{n}\left\|y_{n}\right\|=\lim _{n} \frac{1}{2}\left\|x_{n}+y_{n}\right\|=1$ (see [18], Proposition 5.2.8).

Lemma 7 Let E be a nonempty bounded closed convex subset of a uniformly convex Banach space $X$. If $T: E \rightarrow \mathcal{B C}(X)$ is a nonexpansive mapping, then it is of convex type.

Proof Let $\left\{u_{n}\right\}$ and $\left\{v_{n}\right\}$ be sequences in $E$ such that $\lim _{n} D\left(u_{n}, T u_{n}\right)=\lim _{n} D\left(v_{n}, T v_{n}\right)=0$ and $z_{n}=\frac{1}{2} u_{n}+\frac{1}{2} v_{n}$ for all $n \geq 1$. For each $n$, let $z_{n}^{\prime} \in T z_{n}$ be such that

$$
D\left(z_{n}, T z_{n}\right)-\frac{1}{n} \leq\left\|z_{n}-z_{n}^{\prime}\right\| \leq D\left(z_{n}, T z_{n}\right)
$$

It follows from Proposition 2 that

$$
\begin{aligned}
\underset{n}{\limsup }\left\|u_{n}-z_{n}^{\prime}\right\| & \leq \lim _{n} D\left(u_{n}, T u_{n}\right)+\underset{n}{\limsup }\left\|u_{n}-z_{n}\right\| \\
& =\frac{1}{2} \limsup _{n}\left\|u_{n}-v_{n}\right\| .
\end{aligned}
$$

Similarly, we have

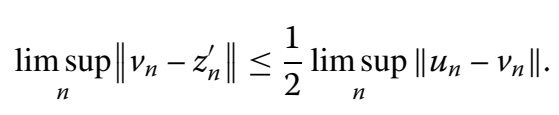

Without loss of generality, we may assume that $\alpha:=\lim _{n}\left\|u_{n}-z_{n}^{\prime}\right\|, \beta:=\lim _{n}\left\|v_{n}-z_{n}^{\prime}\right\|$, and $\gamma:=\lim _{n}\left\|u_{n}-v_{n}\right\|$ do exist. If $\gamma=0$, then we are done. We now assume that $\gamma>0$. Let us consider the following:

$$
\begin{aligned}
\gamma & =\lim _{n}\left\|u_{n}-v_{n}\right\| \\
& =\lim _{n}\left\|\left(u_{n}-z_{n}^{\prime}\right)+\left(z_{n}^{\prime}-v_{n}\right)\right\| \\
& \leq \lim _{n}\left\|u_{n}-z_{n}^{\prime}\right\|+\lim _{n}\left\|z_{n}^{\prime}-v_{n}\right\| \\
& =\alpha+\beta \leq \frac{1}{2} \gamma+\frac{1}{2} \gamma=\gamma .
\end{aligned}
$$

It then follows that $\alpha=\beta=\frac{1}{2} \gamma>0$. In particular,

$$
\begin{aligned}
\lim _{n}\left\|\frac{u_{n}-z_{n}^{\prime}}{\alpha}\right\| & =\lim _{n}\left\|\frac{z_{n}^{\prime}-v_{n}}{\beta}\right\| \\
& =\lim _{n} \frac{1}{2}\left\|\frac{u_{n}-z_{n}^{\prime}}{\alpha}+\frac{z_{n}^{\prime}-v_{n}}{\beta}\right\|=1 .
\end{aligned}
$$

By the uniform convexity of $X$ we have

$$
\lim _{n}\left\|\frac{u_{n}-z_{n}^{\prime}}{\gamma / 2}-\frac{z_{n}^{\prime}-v_{n}}{\gamma / 2}\right\|=\lim _{n}\left\|\frac{u_{n}-z_{n}^{\prime}}{\alpha}-\frac{z_{n}^{\prime}-v_{n}}{\beta}\right\|=0 .
$$


Hence, $\lim _{n}\left\|z_{n}-z_{n}^{\prime}\right\|=\lim _{n}\left\|\frac{1}{2}\left(u_{n}+v_{n}\right)-z_{n}^{\prime}\right\|=0$. This implies that

$$
\lim _{n} D\left(z_{n}, T z_{n}\right) \leq \lim _{n}\left(\left\|z_{n}-z_{n}^{\prime}\right\|+\frac{1}{n}\right)=0
$$

Consequently, $T$ is of convex type.

Theorem 8 Suppose that $E$ is a nonempty bounded closed convex subset of a uniformly convex Banach space $X$. If $T: E \rightarrow \mathcal{B C}(X)$ is a continuous multivalued mapping of convex type, then (A) holds.

Proof For each $r>0$, let $B(r)$ denote the closed ball centered at zero and radius $r$. Set

$$
R:=\left\{r>0: E \cap B(r) \neq \varnothing \text { and } \inf _{x \in E \cap B(r)} D(x, T x)=0\right\} .
$$

It follows from the boundedness of $E$ that $\alpha:=\inf R<\infty$. If $\alpha=0$, then it follows that $0 \in E$ and $0 \in \operatorname{End}(T)$. We now consider the case $\alpha>0$. For each $n \geq 1$, let $x_{n} \in E \cap B(\alpha+1 / n)$ and $D\left(x_{n}, T x_{n}\right) \leq 1 / n$. In particular, $\limsup _{n}\left\|x_{n}\right\| \leq \alpha$ and $\lim _{n} D\left(x_{n}, T x_{n}\right)=0$. If $\left\{x_{n}\right\}$ contains a convergent subsequence, then its limit point is an endpoint of $T$. Suppose that there are a constant $\varepsilon>0$ and a subsequence $\left\{x_{n_{k}}\right\}$ such that $\left\|x_{n_{k}}-x_{n_{k+1}}\right\| \geq \varepsilon$ for all $k \geq 1$. Set $u_{k} \equiv x_{n_{k}}$, $v_{k} \equiv x_{n_{k+1}}$, and $z_{k} \equiv \frac{1}{2} u_{k}+\frac{1}{2} v_{k}$. It follows that $\lim _{k} D\left(u_{k}, T u_{k}\right)=\lim _{k} D\left(v_{k}, T v_{k}\right)=0$. Note that $\limsup _{k}\left\|u_{k}\right\| \leq \alpha$ and $\limsup _{k}\left\|v_{k}\right\| \leq \alpha$. By the uniform convexity of $X$ there exists $\delta>0$ such that $\limsup _{k}\left\|z_{k}\right\| \leq(1-\delta) \alpha<\alpha$. Moreover, it follows from the convexity type of $T$ that $\lim _{n} D\left(z_{n}, T z_{n}\right)=0$. We now obtain a contradiction. The proof is finished.

\subsection{Endpoint results in reflexive spaces with Opial property}

A Banach space $X$ is said to have the Opial property [20] if whenever $\left\{x_{n}\right\}$ is weakly convergent to $x$ and $y \neq x$, it follows that

$$
\limsup _{n}\left\|x_{n}-x\right\|<\limsup _{n}\left\|x_{n}-y\right\|
$$

The following result is related to the demiclosedness property.

Proposition 9 Let $E$ be a nonempty subset of a Banach space $X$ with the Opial property, and $T: E \rightarrow \mathcal{B C}(X)$ be a nonexpansive mapping. If $\left\{u_{n}\right\}$ is a sequence in $E$ such that $D\left(u_{n}, T u_{n}\right) \rightarrow 0$ and $\left\{u_{n}\right\}$ converges weakly to $u \in E$, then $u \in \operatorname{End}(T)$.

Proof Let $u^{\prime} \in T u$. It follows from Proposition 2 that

$$
\left\|u_{n}-u^{\prime}\right\| \leq D\left(u_{n}, T u_{n}\right)+\left\|u_{n}-u\right\|
$$

In particular,

$$
\underset{n}{\limsup }\left\|u_{n}-u^{\prime}\right\| \leq \underset{n}{\lim \sup }\left\|u_{n}-u\right\|
$$

It follows from the Opial property that $u=u^{\prime} \in T u$. This completes the proof.

The following result extends Theorem P2 from $T: E \rightarrow \mathcal{K}(X)$ to $T: E \rightarrow \mathcal{B C}(X)$. 
Theorem 10 Suppose that $E$ is a nonempty bounded closed convex subset of a reflexive Banach space $X$ with the Opial property. If $T: E \rightarrow \mathcal{B C}(X)$ is a nonexpansive mapping, then (A) holds.

Proof Assume that $T$ has an approximate endpoint property. Let $\left\{u_{n}\right\}$ be a sequence in $E$ such that $D\left(u_{n}, T u_{n}\right) \rightarrow 0$. Since $\left\{u_{n}\right\}$ is bounded, there exists a subsequence $\left\{u_{n_{k}}\right\}$ of $\left\{u_{n}\right\}$ such that $\left\{u_{n_{k}}\right\}$ converges weakly to some element $u \in E$. It follows from the preceding proposition that $u \in \operatorname{End}(T)$.

\subsection{Endpoint results in geodesic spaces whose curvature is bounded above}

In this section, we extend both Theorems P3 and P4 in a more general setting, that is, we consider geodesic spaces whose curvature is bounded above. Let us recall relevant definitions and a concept as follows. For more details on the subject, we refer to [21].

For $\kappa \in \mathbb{R}$, let $M_{\kappa}:=\left(M_{\kappa}, d_{\kappa}\right)$ denote the unique simply connected surface (real twodimensional Riemannian manifold) with constant curvature $\kappa$. That is,

$$
M_{\kappa}:= \begin{cases}(1 / \sqrt{\kappa}) \mathbb{S}^{2} & \text { if } \kappa>0 \\ \mathbb{R}^{2} & \text { if } \kappa=0 \\ (1 / \sqrt{-\kappa}) \mathbb{H}^{2} & \text { if } \kappa<0\end{cases}
$$

Here $\mathbb{S}^{2}, \mathbb{R}^{2}$, and $\mathbb{H}^{2}$ represent the two-dimensional sphere, Euclidean space, and hyperbolic space, respectively. In particular,

$$
D_{\kappa}:=\operatorname{diam} M_{\kappa}= \begin{cases}\pi / \sqrt{\kappa} & \text { if } \kappa>0 \\ \infty & \text { if } \kappa \leq 0\end{cases}
$$

A geodesic path joining two elements $x, y$ in a metric space $(X, d)$ is an isometry $c$ : $[0, l] \rightarrow X$, where $d(x, y)=l$, such that $c(0)=x$ and $c(l)=y$. The image of a geodesic path is called a geodesic segment. A metric space for which every two points can be joined by a geodesic segment is called a geodesic space. For any three elements $x, y, z \in X$, a geodesic triangle $\triangle(x, y, z)$ is the union of three geodesic segments joining each two of them. Since there may be more than one geodesic segment joining each two points, the triangle $\triangle(x, y, z)$ depends on the geodesic paths we choose. We say that a geodesic triangle $\Delta(x, y, z)$, where $x, y, z \in X$ and $d(x, y)+d(y, z)+d(z, x)<2 D_{\kappa}$, satisfies the $\operatorname{CAT}(\kappa)$ inequality if there exists corresponding three points $\bar{x}, \bar{y}, \bar{z} \in M_{\kappa}$ such that

- $d(x, y)=d_{M_{\kappa}}(\bar{x}, \bar{y}), d(y, z)=d_{M_{k}}(\bar{y}, \bar{z})$, and $d(x, z)=d_{M_{\kappa}}(\bar{x}, \bar{z})$;

- $d(p, q) \leq d_{M_{\kappa}}(\bar{p}, \bar{q})$ for all $p, q \in \triangle(x, y, z)$ and $\bar{p}, \bar{q} \in \triangle(\bar{x}, \bar{y}, \bar{z})$.

A geodesic metric space $(X, d)$ is a $\operatorname{CAT}(\kappa)$ space if every geodesic triangle $\Delta$ in $X$ with perimeter less than $2 D_{k}$ satisfies the $\operatorname{CAT}(\kappa)$ inequality. If $X$ is a $\operatorname{CAT}(\kappa)$ space, then it is $D_{\kappa}$-uniquely geodesic, that is, there exists a unique geodesic path joining $x$ and $y$ for all $x, y \in X$ with $d(x, y)<D_{\kappa}$. In this case, we denote the unique geodesic segment joining $x$ and $y$ by $[x, y]$, and the element $z \in[x, y]$ satisfying $d(z, x)=(1-t) d(x, y)$ and $d(z, y)=t d(x, y)$ for some $t \in[0,1]$ is also denoted by $t x \oplus(1-t) y$. A subset $Y$ of a CAT $(\kappa)$ space is $D_{\kappa}$-convex if $[x, y] \subset Y$ for all $x, y \in Y$ with $d(x, y)<D_{\kappa}$. 
Note that in this study we can consider only CAT(1) spaces because all the results can be easily extended to $\operatorname{CAT}(\kappa)$ spaces with $\kappa>0$ by resizing the space. Moreover, every $\operatorname{CAT}\left(\kappa_{1}\right)$ space is a $\operatorname{CAT}\left(\kappa_{2}\right)$ space whenever $\kappa_{1} \leq \kappa_{2}$.

Lemma 11 ([22], Lemma 3.1) Let $\triangle(x, y, z)$ be a geodesic triangle in a CAT(1) space such that $d(x, y)+d(x, z)+d(y, z)<2 \pi$, and let $w \in[x, y]$. Then

$$
\cos d(w, z) \sin d(x, y) \geq \cos d(x, z) \sin d(y, w)+\cos d(y, z) \sin d(x, w) .
$$

Theorem 12 Let $E$ be a nonempty convex subset of a complete CAT(1) space X. If $T: E \rightarrow$ $\mathcal{B C}(X)$ is a nonexpansive mapping, then $\operatorname{End}(T)$ is $\pi$-convex.

Proof Let $u, v \in \operatorname{End}(T)$ be such that $0<d(u, v)<\pi$. Let $w \in[u, v]$. Note that $w \in E$. We show that $w \in \operatorname{End}(T)$. To see this, let $w^{\prime} \in T w$. It follows from Proposition 2 that

$$
d\left(u, w^{\prime}\right) \leq d(u, w) \quad \text { and } \quad d\left(v, w^{\prime}\right) \leq d(v, w)
$$

Moreover, we have the following:

$$
\begin{aligned}
\sin d(u, v) & \geq \cos d\left(w, w^{\prime}\right) \sin d(u, v) \\
& \geq \cos d\left(u, w^{\prime}\right) \sin d(v, w)+\cos d\left(v, w^{\prime}\right) \sin d(u, w) \\
& \geq \cos d(u, w) \sin d(v, w)+\cos d(v, w) \sin d(u, w) \\
& =\sin (d(u, w)+d(v, w)) \\
& =\sin d(u, v)
\end{aligned}
$$

In particular, $\cos d\left(w, w^{\prime}\right)=1$. This implies that $d\left(w, w^{\prime}\right)=0$, that is, $w=w^{\prime} \in T w$.

Let $E$ be a nonempty subset of a CAT(1) space $X$ such that $d(u, v)<\pi$ for all $u, v \in E$. Analogously to Definition 6, we introduce the following concept: $T: E \rightarrow \mathcal{B C}(X)$ is of convex type if $\lim _{n} D\left(z_{n}, T z_{n}\right)=0$ whenever $\left\{u_{n}\right\}$ and $\left\{v_{n}\right\}$ are sequences in $E$ such that $\lim _{n} D\left(u_{n}, T u_{n}\right)=\lim _{n} D\left(v_{n}, T v_{n}\right)=0$ and $z_{n}=\frac{1}{2} u_{n} \oplus \frac{1}{2} v_{n}$ for all $n \geq 1$. It is shown in the following theorem that this class of mappings includes all nonexpansive ones.

Theorem 13 Let $E$ be a nonempty convex subset of a complete CAT(1) space $X$ such that $\operatorname{diam}(E)<\pi$. If $T: E \rightarrow \mathcal{B C}(X)$ is nonexpansive, then it is of convex type.

Proof Let $\left\{u_{n}\right\}$ and $\left\{v_{n}\right\}$ be sequences in $E$ such that $\lim _{n} D\left(u_{n}, T u_{n}\right)=\lim _{n} D\left(v_{n}, T v_{n}\right)=0$ and $z_{n}=\frac{1}{2} u_{n} \oplus \frac{1}{2} v_{n}$ for all $n \geq 1$. We show that $\lim _{n} D\left(z_{n}, T z_{n}\right)=0$. To see this, let $z_{n}^{\prime} \in T z_{n}$ be such that

$$
D\left(z_{n}, T z_{n}\right)-\frac{1}{n} \leq d\left(z_{n}, z_{n}^{\prime}\right) .
$$

It follows from Proposition 2 that

$$
\limsup _{n} d\left(u_{n}, z_{n}^{\prime}\right) \leq \lim _{n} D\left(u_{n}, T u_{n}\right)+\underset{n}{\limsup } d\left(u_{n}, z_{n}\right)=\frac{1}{2} \limsup _{n} d\left(u_{n}, v_{n}\right) .
$$


Similarly, we have

$$
\limsup _{n} d\left(v_{n}, z_{n}^{\prime}\right) \leq \frac{1}{2} \limsup _{n} d\left(u_{n}, v_{n}\right)
$$

Passing to subsequences, we may assume that $\alpha:=\lim _{n} d\left(u_{n}, z_{n}^{\prime}\right), \beta:=\lim _{n} d\left(v_{n}, z_{n}^{\prime}\right)$, and $\gamma:=\lim _{n} d\left(u_{n}, v_{n}\right)$ exist. Note that $\alpha \leq \frac{1}{2} \gamma$ and $\beta \leq \frac{1}{2} \gamma$. If $\gamma=0$, then $\lim _{n} d\left(z_{n}, z_{n}^{\prime}\right) \leq$ $\lim _{n}\left(d\left(z_{n}, u_{n}\right)+d\left(u_{n}, z_{n}^{\prime}\right)\right) \leq 2 \lim _{n} d\left(z_{n}, u_{n}\right)+\lim _{n} D\left(u_{n}, T u_{n}\right)=\lim _{n} d\left(u_{n}, v_{n}\right)+\lim _{n} D\left(u_{n}\right.$, $\left.T u_{n}\right)=0$. This implies that $\lim _{n} D\left(z_{n}, T z_{n}\right) \leq \lim _{n}\left(d\left(z_{n}, z_{n}^{\prime}\right)+\frac{1}{n}\right)=0$. We now assume that $\gamma>0$. Now let us consider the following:

$$
\begin{aligned}
& \sin \gamma \geq \limsup _{n} \cos d\left(z_{n}, z_{n}^{\prime}\right) \sin \gamma \\
& =\limsup _{n} \cos d\left(z_{n}, z_{n}^{\prime}\right) \sin d\left(u_{n}, v_{n}\right) \\
& \geq \lim \sup \left(\cos d\left(u_{n}, z_{n}^{\prime}\right) \sin d\left(z_{n}, v_{n}\right)+\cos d\left(v_{n}, z_{n}^{\prime}\right) \sin d\left(z_{n}, u_{n}\right)\right) \\
& =\cos \alpha \sin \frac{1}{2} \gamma+\cos \beta \sin \frac{1}{2} \gamma \\
& \geq \cos \frac{1}{2} \gamma \sin \frac{1}{2} \gamma+\cos \frac{1}{2} \gamma \sin \frac{1}{2} \gamma=\sin \gamma \text {. }
\end{aligned}
$$

In particular, since $\gamma<\pi$, we have $\lim \sup _{n} \cos d\left(z_{n}, z_{n}^{\prime}\right)=1$. This implies that $\lim _{n} d\left(z_{n}, z_{n}^{\prime}\right)=$ 0 , that is, $\lim _{n} D\left(z_{n}, T z_{n}\right) \leq \lim _{n}\left(d\left(z_{n}, z_{n}^{\prime}\right)+\frac{1}{n}\right)=0$. The proof is finished.

The proof of the following result is very similar to that of Theorem 8 .

Theorem 14 Let $E$ be a nonempty closed convex subset of a complete CAT(1) space $X$ such that $\operatorname{diam}(E)<\pi$ and $d(x, y)+d(y, z)+d(z, x)<2 \pi$ for all $x, y, z \in E$. If $T: E \rightarrow \mathcal{B C}(X)$ is of convex type and continuous, then (A) holds.

Proof Assume that $T$ has the approximate endpoint property. Let $x_{0} \in E$ be fixed and $B(r)$ denote the closed ball centered at $x_{0}$ and radius $r>0$. Set

$$
R:=\left\{r>0: E \cap B(r) \neq \varnothing \text { and } \inf _{x \in E \cap B(r)} D(x, T x)=0\right\} .
$$

Note that $\alpha:=\inf R<\infty$. If $\alpha=0$, then it follows that $x_{0} \in \operatorname{End}(T)$. We now consider the case $\alpha>0$. For each $n \geq 1$, let $x_{n} \in E \cap B(\alpha+1 / n)$ and $D\left(x_{n}, T x_{n}\right) \leq 1 / n$. In particular, $\lim \sup _{n} d\left(x_{n}, x_{0}\right) \leq \alpha$ and $\lim _{n} D\left(x_{n}, T x_{n}\right)=0$. If $\left\{x_{n}\right\}$ contains a convergent subsequence $\left\{x_{n_{k}}\right\}$ such that $\left\{x_{n_{k}}\right\}$ converges to some element $x \in E$, then $x \in \operatorname{End}(T)$. On the other hand, we suppose that there are a constant $\varepsilon>0$ and a subsequence $\left\{x_{n_{k}}\right\}$ such that $d\left(x_{n_{k}}, x_{n_{k+1}}\right) \geq \varepsilon$ for all $k \geq 1$. Set $u_{k} \equiv x_{n_{k}}, v_{k} \equiv x_{n_{k+1}}$, and $z_{k} \equiv \frac{1}{2} u_{k} \oplus \frac{1}{2} v_{k}$. It follows that $\lim _{k} D\left(u_{k}, T u_{k}\right)=\lim _{k} D\left(v_{k}, T v_{k}\right)=0$. Since $T$ is of convex type, we have $\lim _{k} D\left(z_{k}, T z_{k}\right)=0$. Note that $\limsup _{k} d\left(u_{k}, x_{0}\right) \leq \alpha, \lim \sup _{k} d\left(v_{k}, x_{0}\right) \leq \alpha$, and $\varepsilon \leq d\left(u_{k}, v_{k}\right) \leq \operatorname{diam}(E)<\pi$ for 
all $k \geq 1$. Since $d\left(x_{0}, u_{k}\right)+d\left(u_{k}, v_{k}\right)+d\left(v_{k}, x_{0}\right)<2 \pi$, we have

$$
\begin{aligned}
& \cos d\left(z_{k}, x_{0}\right) \sin d\left(u_{k}, v_{k}\right) \\
& \quad \geq \cos d\left(u_{k}, x_{0}\right) \sin d\left(v_{k}, z_{k}\right)+\cos d\left(v_{k}, x_{0}\right) \sin d\left(u_{k}, z_{k}\right) \\
& \quad=\left(\cos d\left(u_{k}, x_{0}\right)+\cos d\left(v_{k}, x_{0}\right)\right) \sin \frac{1}{2} d\left(u_{k}, v_{k}\right)
\end{aligned}
$$

In particular,

$$
\begin{aligned}
\cos d\left(z_{k}, x_{0}\right) \geq & \left(\cos d\left(u_{k}, x_{0}\right)+\cos d\left(v_{k}, x_{0}\right)\right) \frac{\sin \frac{1}{2} d\left(u_{k}, v_{k}\right)}{\sin d\left(u_{k}, v_{k}\right)} \\
& =\frac{\cos d\left(u_{k}, x_{0}\right)+\cos d\left(v_{k}, x_{0}\right)}{2 \cos \frac{1}{2} d\left(u_{k}, v_{k}\right)}
\end{aligned}
$$

This implies that

$$
\liminf _{k} \cos d\left(z_{k}, x_{0}\right) \geq \frac{\cos \alpha}{\cos \frac{1}{2} \varepsilon}>\cos \alpha .
$$

Since $t \mapsto \cos t$ is strictly decreasing on $(0, \pi)$, there exists $\delta>0$ such that

$$
\underset{k}{\lim \sup } d\left(z_{k}, x_{0}\right) \leq \alpha-\delta<\alpha,
$$

which is a contradiction. The proof is finished.

The following result improves that of Espínola and Fernández-León [23]. It is clear that the condition $\operatorname{diam}(E)<\pi / 2$ implies $\operatorname{diam}(E)<\pi$ and $d(x, y)+d(y, z)+d(z, x)<2 \pi$ for all $x, y, z \in E$. In the proof above, we do not use the modulus of convexity of $\mathbb{S}^{2}$ endowed with the spherical distance as was the case in [23].

Corollary 15 Let E be a nonempty closed convex subset of a complete CAT(1) space $X$ such that $\operatorname{diam}(E)<\pi$ and $d(x, y)+d(y, z)+d(z, x)<2 \pi$ for all $x, y, z \in E$. Suppose that $T: E \rightarrow X$ is of convex type and is continuous. Then $\operatorname{Fix}(T) \neq \varnothing$ if and only if $\inf \{d(x, T x): x \in E\}=0$.

\section{Competing interests}

The author declares that he has no competing interests.

\section{Acknowledgements}

The author would like to thank the two referees for their comments and suggestions. This work is partially supported by the Research Center for Environmental and Hazardous Substance Management, Khon Kaen University.

Received: 29 September 2015 Accepted: 8 April 2016 Published online: 16 April 2016

\section{References}

1. Corley, HW: Some hybrid fixed point theorems related to optimization. J. Math. Anal. Appl. 120(2), 528-532 (1986)

2. Itoh, S, Takahashi, W: Single-valued mappings, multivalued mappings and fixed-point theorems. J. Math. Anal. Appl. 59(3), 514-521 (1977)

3. Klein, E, Thompson, AC: Theory of Correspondences. Including Applications to Mathematical Economics. Canadian Mathematical Society Series of Monographs and Advanced Texts. A Wiley-Interscience Publication. Wiley, New York (1984)

4. Kojima, M, Yamamoto, Y: A unified approach to the implementation of several restart fixed point algorithms and a new variable dimension algorithm. Math. Program. 28(3), 288-328 (1984)

5. Llorens-Fuster, E: Multivalued nonexpansive mappings with an almost convex displacement function. J. Nonlinear Convex Anal. 16(9), 1835-1845 (2015) 
6. Pietramala, P: Convergence of approximating fixed points sets for multivalued nonexpansive mappings. Comment. Math. Univ. Carol. 32(4), 697-701 (1991)

7. Reich, S: Fixed point theorems for set-valued mappings. J. Math. Anal. Appl. 69(2), 353-358 (1979)

8. Todd, MJ: The Computation of Fixed Points and Applications. Lecture Notes in Economics and Mathematical Systems, vol. 124. Springer, Berlin (1976)

9. Ko, HM: Fixed point theorems for point-to-set mappings and the set of fixed points. Pac. J. Math. 42, 369-379 (1972)

10. Belluce, LP, Kirk, WA: Some fixed point theorems in metric and Banach spaces. Can. Math. Bull. 12, 481-491 (1969)

11. Montagnana, M, Vignoli, A: On quasiconvex mappings and fixed point theorems. Boll. Unione Mat. Ital. (4) 4, 870-878 (1971)

12. Daneš, J: Fixed point theorems, Nemyckii and Uryson operators, and continuity of nonlinear mappings. Comment. Math. Univ. Carol. 11, 481-500 (1970)

13. García-Falset, J, Llorens-Fuster, E, Sims, B: Fixed point theory for almost convex functions. Nonlinear Anal. 32(5), 601-608 (1998)

14. Llorens-Fuster, E: Set-valued $\alpha$-almost convex mappings. J. Math. Anal. Appl. 233(2), 698-712 (1999)

15. Panyanak, B: Endpoints of multivalued nonexpansive mappings in geodesic spaces. Fixed Point Theory Appl. 2015 , 147 (2015)

16. Lim, TC: Remarks on some fixed point theorems. Proc. Am. Math. Soc. 60, 179-182 (1976)

17. Clarkson, JA: Uniformly convex spaces. Trans. Am. Math. Soc. 40(3), 396-414 (1936)

18. Megginson, RE: An Introduction to Banach Space Theory. Graduate Texts in Mathematics, vol. 183. Springer, New York (1998)

19. Kirk, WA: Nonexpansive mappings in metric and Banach spaces. Rend. Semin. Mat. Fis. Milano 51, 133-144 (1981)

20. Opial, Z: Weak convergence of the sequence of successive approximations for nonexpansive mappings. Bull. Am. Math. Soc. 73, 591-597 (1967)

21. Bridson, MR, Haefliger, A: Metric Spaces of Non-positive Curvature. Grundlehren der Mathematischen Wissenschaften [Fundamental Principles of Mathematical Sciences], vol. 319. Springer, Berlin (1999)

22. Kimura, Y, Satô, K: Halpern iteration for strongly quasinonexpansive mappings on a geodesic space with curvature bounded above by one. Fixed Point Theory Appl. 2013, 7 (2013)

23. Espínola, R, Fernández-León, A: CAT(k)-Spaces, weak convergence and fixed points. J. Math. Anal. Appl. 353(1), $410-427(2009)$

\section{Submit your manuscript to a SpringerOpen ${ }^{\ominus}$ journal and benefit from:}

- Convenient online submission

Rigorous peer review

- Immediate publication on acceptance

- Open access: articles freely available online

- High visibility within the field

- Retaining the copyright to your article 